\title{
Évolutions récentes du système éducatif de l'Ouzbékistan
}

Lisa Bydanova et Daniyor Rozmetov

\section{OpenEdition}

12 Journals

Édition électronique

URL : http://journals.openedition.org/ries/3671

DOI : 10.4000/ries.3671

ISSN : 2261-4265

Éditeur

Centre international d'études pédagogiques

Édition imprimée

Date de publication : 15 avril 2014

Pagination : 28-33

ISBN : 978-2-85420-603-6

ISSN : $1254-4590$

Référence électronique

Lisa Bydanova et Daniyor Rozmetov, "Évolutions récentes du système éducatif de l'Ouzbékistan », Revue internationale d'éducation de Sèvres [En ligne], 65 I avril 2014, mis en ligne le 15 avril 2016,

consulté le 08 janvier 2020. URL : http://journals.openedition.org/ries/3671 ; DOI : 10.4000/ries.3671

Ce document a été généré automatiquement le 8 janvier 2020

(c) Tous droits réservés 


\title{
Évolutions récentes du système éducatif de l'Ouzbékistan
}

\author{
Lisa Bydanova et Daniyor Rozmetov
}

1 L'Ouzbékistan est une ancienne république soviétique située en Asie centrale. C'est le seul pays de la région à partager ses frontières avec tous les pays de la région: le Kazakhstan au nord, le Kirghizistan et le Tadjikistan à l'est, l'Afghanistan au sud et le Turkménistan au sud-ouest, le long du fleuve Amou-Daria. Avec une population de plus de 29 millions d'habitants sur un territoire de $447400 \mathrm{~km}^{2}$, l'Ouzbékistan est le pays le plus peuplée d'Asie centrale et le troisième pays en superficie. Environ $80 \%$ de la population est ouzbek. Parmi les minorités, plus de 120 nationalités et ethnies cohabitent dans le pays, et notamment les Russes (5,5\%), les Tadjiks (5\%), les Kazakhs (3\%), les Karakalpaks (2,5\%), les Tatares (1,5\%). La langue officielle est l'ouzbek (langue appartenant à la branche turcique de la famille des langues altaïques), la majorité de la population est musulmane ${ }^{1}$.

2 L'économie du pays repose sur l'exportation des matières premières (or, uranium et gaz), et surtout sur le secteur de l'agriculture qui emploie $40 \%$ de la population active et est marqué par sa spécialisation dans la culture de coton $\left(18 \% \mathrm{du}\right.$ PIB, $5^{\mathrm{e}}$ producteur et 2e exportateur mondial). Depuis l'époque soviétique, l'Ouzbékistan a hérité d'un important tissu industriel (métallurgie, chimie, aéronautique) que l'État s'emploie à moderniser et qui connaît quelques succès (importants investissements récents de compagnies étrangères dans le domaine de l'automobile telles que General Motors, Mercedes-Benz, MAN, Koç Holding). La part de la population rurale s'élève à $63 \%$ et $41 \%$ de la population est âgée de moins de 17 ans.

3 L'éducation et l'enseignement occupent en Ouzbékistan, comme dans toutes les républiques anciennement soviétiques, une place importante. Le niveau d'alphabétisation dans le pays est proche de $100 \%$ et les dépenses publiques en éducation sont particulièrement élevées. En 2009, elles s'élevaient à 10,8\%, ce qui est exceptionnel, comparé à d'autres pays de la région et à la plupart des pays occidentaux. Selon l'indice du développement humain (IDH), le pays est classé en 114e position, 
faisant partie du groupe des pays à développement humain moyen. En 2013, l'Ouzbékistan a rejoint l'initiative du Partenariat mondial pour l'éducation.

\section{La structure du système éducatif et la base législative}

4 L'article 41 de la Constitution de la République d'Ouzbékistan, adopté le 8 décembre 1992, garantit à tous ses citoyens l'accès gratuit à l'enseignement ${ }^{2}$. Les réformes du système éducatif, qui se sont imposées suite à l'indépendance et à l'abandon du système de planification, ont été mises en place avec la Loi sur l'éducation ${ }^{3}$ et le Programme national de la formation des cadres, votés en 1997. Ces actes juridiques prévoyaient une nouvelle approche du management et du financement de l'éducation, une refonte des programmes d'enseignement et une réorganisation de la structure de l'enseignement. La Loi sur l'éducation visait à affirmer le caractère humaniste, démocratique et laïc de l'enseignement. Le Programme national de la formation des cadres insistait sur la nécessité de libérer le système éducatif des anciennes approches idéologiques et de créer un système national pour former des cadres de haut niveau, avec une culture et une éducation répondant aux standards mondiaux semblables à ceux des pays développés et démocratiques ${ }^{4}$. En 2007, la Loi sur l'éducation a été révisée pour insister sur les droits des enfants et rappeler le principe de la gratuité de l'éducation pour tous les enfants. En 2004, le Programme national pour le développement de l'éducation 2004-2009 mettait l'accent sur la qualité de l'éducation.

5 En septembre 1993, le pays a adopté la Loi sur l'adoption du nouvel alphabet ouzbek, basé sur l'alphabet latin ${ }^{5}$. En effet, le peuple habitant l'Ouzbékistan écrivait en caractères arabes depuis l'arrivée des Arabes au VIII siècle. Entre 1929 et 1940, le pays, qui faisait partie de l'Union soviétique, avait adopté l'alphabet latin, mais en 1940, l'Union soviétique déclara le cyrillique alphabet de toute l'Union. De ce fait, les avis sont partagés aujourd'hui en Ouzbékistan sur le nouveau changement d'alphabet survenu après l'indépendance.

La structure du système éducatif actuel est la suivante ${ }^{6}$ :

- l'éducation préscolaire (jusqu'à 6-7 ans);

- l'enseignement secondaire général: neuf années d'études (7-15 ans), dont quatre de primaire (7-10 ans) ;

- l'enseignement secondaire spécialisé et la formation technique et professionnelle: trois années d'études (15-18 ans); l'enseignement secondaire spécialisé est proposé dans des lycées généraux spécialisés dans les matières scientifiques; les lycées professionnels, appelés « collèges », offrent des formations techniques et professionnelles;

- l'enseignement supérieur divisé en trois niveaux : les programmes de Bakalavr (équivalent de la licence, avec quatre années d'études), de Magistr (équivalent du master, deux années d'études) et de Fanlar Nomzodi (équivalent du doctorat, avec trois années d'études); ces programmes sont proposés par trois types d'établissements d'enseignement supérieur (universités, instituts et académies); l'accès à l'enseignement supérieur s'effectue par le biais d'un test national, qui est organisé le $1^{\text {er }}$ août chaque année, et comprend trois matières, avec 36 items chacune.

6 Dans l'enseignement général, on compte 4,5 millions d'élèves scolarisés dans 9765 écoles de l'enseignement secondaire dont 8742 en langue ouzbèke, 377 en karakalpak, 848 en russe, 417 en kazakh, 256 en tadjik, 60 en kirghiz et 43 en turkmène ${ }^{7}$. L'enseignement supérieur accueille 289208 étudiants, répartis entre $59^{8}$ établissements 
d'enseignement supérieurs, dont six annexes d'universités étrangères (Université d'État du pétrole et du gaz de la Russie de Gubkin, Académie économique de la Russie de Plekhanov, Université d'État de la Russie de Lomonosov, Institut de développement du management de Singapour, Université Polytechnique de Turin et Université internationale Westminster de Londres). Une filiale de l'université coréenne Inha doit ouvrir prochainement ${ }^{9}$. Avant l'indépendance, les trois quarts des établissements d'enseignement supérieur étaient situés dans quatre grandes villes du pays (Tachkent, Samarkand, Andijan et Bukhara). Les réformes ont permis une meilleure répartition géographique des établissements, et dorénavant chacune des quatorze régions ${ }^{10}$ possède une université nationale. Bien que l'Ouzbékistan ne fasse pas partie du processus de Bologne, la mobilité étudiante y est relativement importante. En 2011, $8,42 \%$ des étudiants ${ }^{11}$ effectuaient un séjour de mobilité à l'étranger. Les cinq premiers pays d'accueil sont la Russie, le Kirghizstan, le Kazakhstan, le Tadjikistan et l'Allemagne.

\section{Gouvernance et financements}

7 Malgré quelques tentatives récentes de décentralisation, le système éducatif ouzbek reste centralisé, comme sous la période soviétique. Les deux ministères en charge du secteur éducatif, le ministère de l'éducation nationale (MEN) et le ministère de l'enseignement supérieur et secondaire spécialisé (MESS), définissent les principes et l'organisation générale du système éducatif, élaborent les maquettes des formations pour l'ensemble du territoire national et établissent les grilles salariales des enseignants. Le MEN est responsable de la formation des enseignants de tous les niveaux, dans les six instituts de formation initiale du pays. Les établissements d'enseignement supérieur sont sous la tutelle du MESS ou d'autres ministères sectoriels.

8 Les présidents des universités (« recteurs ») sont nommés par le Conseil des ministres et il n'existe pas d'établissements de formation de droit privé ${ }^{12}$. Tous les établissements éducatifs appartiennent à l'État, qui finance l'ensemble des frais pour l'enseignement général (70\% des dépenses publiques en éducation sont destinées à ce niveau) et $50 \%$ du budget des universités. Ces dernières bénéficient d'une certaine autonomie financière, qui leur permet d'attirer des financements venant d'autres sources, dont la majeure partie sont les frais d'inscription des étudiants qui n'ont pas obtenu les «places» financées par l'État. En 2009-2010, le quota des places prises en charge par l'État (ce qui implique la gratuité des frais d'inscription et une bourse pour couvrir les frais de vie) s'élevait à 19755 sur 57305 au total, soit $34 \%$, pour les programmes de Bakalavr, et 1559 sur 5647 , soit $28 \%$, pour les programmes de Magistr. Sans l'aide de l'État, les frais d'inscription varient entre 700 et 1500 euro pour une année d'études.

Les frais d'inscription élevés (le PIB/tête étant de 2600 euro en 2012) ne dissuadent pas la demande privée pour le secteur de l'enseignement supérieur, bien au contraire. Le nombre d'étudiants a été multiplié par 1,5 entre 2000 et 2010. Malgré cela, le taux de participation dans l'enseignement supérieur reste largement inférieur à la moyenne de la région (en 2005, il s'élevait à 15 contre 37 en moyenne pour l'Asie centrale et 41 pour les pays de la $\left.\mathrm{CEI}^{13}\right)$. 


\section{Défis actuels}

10 Depuis l'accession du pays à l'indépendance, le système éducatif ouzbek a fait face à de nombreux problèmes. Le nouvel État et ses organes de gouvernance et de planification renouvelés devaient d'une part assurer un financement suffisant de l'ensemble du système, à la hauteur des moyens déployés pendant la période soviétique et, d'autre part, opérer un changement structurel et idéologique complet. Le gouvernement a opté pour une transition graduelle et pour le maintien des acquis du système précédent, autant que possible. Ainsi, on note aujourd'hui des performances de bon niveau en termes d'accès à l'enseignement général et d'égalité des genres; les taux de scolarisation et d'alphabétisation avoisinent $100 \%$, l'éducation gratuite et obligatoire au niveau de l'enseignement général est préservée.

11 Sur cette base, le pays a cherché à renforcer la qualité de l'éducation, à améliorer son adéquation au monde du travail, et à correspondre aux tendances internationales actuelles. Le pays s'ouvre donc progressivement aux coopérations internationales, notamment avec les États-Unis et les pays européens. Les études dans ces pays ou dans les établissements de formation ouverts à leur initiative en Ouzbékistan jouissent d'une bonne image. Y étudier est considéré comme prestigieux et les réformes en cours sont inspirées de leurs modèles.

12 En outre, le secteur éducatif devient un levier important de la construction nationale. La question du passage du russe, omniprésent pendant la période soviétique, à la langue nationale comme langue d'enseignement est au cœur de cet enjeu. Elle rencontre deux difficultés majeures : produire de nouveaux manuels et disposer d'un personnel enseignant qualifié.

13 Il s'agissait en effet non seulement de "traduire» tous les ouvrages de l'idéologie communiste vers celle de l'identité nationale, mais de changer d'alphabet. Cette tâche difficile avait en outre des conséquences lourdes. En effet, tous les savoir-faire et connaissances du pays depuis 1940, comme ceux de l'Union soviétique, étaient rédigés en cyrillique. Mais le rejet de l'ancien régime et la volonté de se faire une place sur la scène internationale étaient si forts que le pays a décidé de supporter le prix à payer pour ce changement d'alphabet.

14 La deuxième difficulté concerne la formation des enseignants : il fallait, d'une part, faire évoluer les mentalités des enseignants qui, pour la plupart, avaient été formés à l'époque soviétique et, d'autre part, moderniser les compétences et les connaissances professionnelles de ces personnels et les rendre compatibles avec les standards internationaux. L'apparition de "l'idéologie nationale de l'indépendance ${ }^{14}$ était censée libérer les enseignants de l'idéologie communiste et renforcer la nouvelle fierté nationale. Pour assurer une formation continue de haut niveau, des coopérations internationales et des échanges bilatéraux ont été mis en place avec les grands établissements de formation du monde entier. De l'importance particulière de cet enjeu témoigne le Programme national de la formation des cadres adopté en 1997 et construit en trois étapes (1997-2001; 2001-2005 ; 2005-période actuelle).

Parmi les autres difficultés auxquelles est confronté aujourd'hui le système éducatif ouzbek, on pourrait citer :

- une faible couverture de la population par l'enseignement préscolaire ; 
- le besoin de rénovation des équipements scolaires restés sans maintenance depuis la fin de l'époque soviétique et pendant la période de transition ;

- le problème de l'attractivité du métier de l'enseignement auquel ont contribué des salaires faibles et de médiocres conditions de travail depuis les quinze dernières années ;

- le besoin de renforcer la qualité desapprentissages et l'adéquation du système d'enseignement et de formation avec les besoins du marché du travail.

Le taux de scolarisation des enfants de moins de 7 ans est très bas en Ouzbékistan. Il ne représente que $19,4 \%$, de l'ensemble du groupe d'âge, soit 642487 élèves répartis dans près de 6865 établissements préscolaires. Des mesures ont été mises en place pour pallier cette insuffisance. Il existe, par exemple, des classes préparatoires spécifiques, parfois de droit privé, qui aident à préparer les enfants n'ayant pu bénéficier de l'éducation préscolaire. L'association publique nationale Farzandim-jigarbandim («Mon enfant, le mien ») a été créé à la suite du décret $n^{\circ} 68 \mathrm{du}$ Conseil des ministres, en février 2001. Cette organisation dispose d'antennes dans six régions. Son but est d'aider les parents à préparer eux-mêmes leurs enfants pour l'école primaire. Ces filiales régionales assurent également la formation préscolaire des enfants issus de familles pauvres.

17 La qualité de l'éducation, notamment des connaissances et compétences, reste difficile à apprécier, car les données disponibles ne permettent pas cette analyse. En effet, l'Ouzbékistan ne participe pas aux grandes enquêtes internationales (contrairement aux pays voisins comme le Kazakhstan, par exemple). L'étude de la Banque mondiale intitulée "Compétences et pas uniquement les diplômes » (Skills, Not Just Diplomas) conduite en 2008, montre que le pourcentage des entreprises du pays qui déclare comme un frein « important » ou « très important » pour leurs activités le manque de compétences du personnel oscille entre 30 et $40 \%$.

\section{BIBLIOGRAPHIE}

Campus France (2013) : Fiche pays Ouzbékistan, novembre 2013.

Données sur l'aménagement linguistique du monde, Université de Laval : http:// www.axl.cefan.ulaval.ca/asie/ouzbekistan.htm.

KURBANOVA D. (2008) : « Le Développement de l'éducation. Rapport national de la République d'Ouzbékistan ", Commission nationale de la République d'Ouzbékistan pour l'UNESCO, 2005.

MURTHI M. \& SONDERGAARD L. (2010): “Skills, Not Just Diplomas: The Path for Education Reforms in Europe and Central Asia", mai 2010, vol. 20.

Unicef (2008) : Country profile.

\section{NOTES}

1. Source : CIA Factbook : https://www.cia.gov/index.html 
2. Constitution de la République d'Ouzbékistan: www.lex.uz/pages/getpage.aspx ? lact_id $=20596$

3. Loi de la République d'Ouzbékistan sur l'éducation: www.lex.uz/pages/GetAct.aspx ? lact_id $=16188$

4. Site officiel du ministère de l'éducation nationale : http://webbogcha.uzedu.uz/index.php? page=stat.php

5. Loi de la République d'Ouzbékistan sur l'adoption du nouvel alphabet ouzbek, basé sur l'alphabet latin : http://www.lex.uz/pages/getpage.aspx?lact_id=112286

6. Programme national de l'Ouzbékistan de la formation des cadres : http://www.lex.uz/pages/ GetAct.aspx?lact_id=48401

7. Site officiel du ministère de l'éducation nationale: www. uzedu.uz/uzb/info/parents/ (données 2012-2013)

8. Site d'information ouzbek sur l'éducation: www.ziyonet.uz/uzc/education/system/ highschool/

9. www.inha.edu

10. L'Ouzbékistan est une république divisée en douze régions (viloyat), auxquelles s'ajoutent la capitale, Tashkent, qui dispose d'un-statut spécial, et la république autonome du Karakalpakstan, qui s'étend sur $164900 \mathrm{~km}^{2}$ (soit près de $37 \%$ du territoire national), à l'ouest de la mer d'Aral.

11. Données de la fiche pays Campus France, novembre 2013

12. Les annexes des universités étrangères sont également des entités de droit public, qui opèrent sur la base des accords intergouvernementaux. Par exemple, l'Université polytechnique de Turin à Tashkent, créée en 2009 sur la base d'un accord entre le ministère de l'enseignement supérieur et secondaire spécialisé de l'Ouzbékistan et l'Université polytechnique de Turin en Italie, est dans l'obligation de tenir compte des standards éducatifs nationaux en Ouzbékistan, bien que les diplômes délivrés soient des diplômes de cette université italienne.

13. Voir la fiche pays de l'Unicef, 2008.

14. Portail d'information et de sources écrites (en ouzbek) : http://www.ziyouz.com/index.php? option=com_remository\&Itemid $=57 \&$ func $=$ fileinfo $\&$ id $=2301$

\section{INDEX}

Mots-clés : financement, politique éducative, système éducatif

Index géographique : Ouzbékistan

Keywords : financing, educational policy, educational system

Palabras claves : financiación, política educacional, sistema educativo

\section{AUTEURS}

\section{LISA BYDANOVA}

Chargée de programmes au Centre international d'études pédagogiques (CIEP, France). Diplômée de l'Université de Bourgogne (IREDU) en sociologie et économie de l'éducation, elle travaille sur le renforcement des capacités des acteurs éducatifs de l'enseignement supérieur (la garantie de la 
qualité dans l'enseignement supérieur et le processus de Bologne) et la formation technique et professionnelle dans les anciens pays de l'URSS (Kazakhstan, Arménie et Géorgie).

\section{DANIYOR ROZMETOV}

Daniyor Rozmetov est doctorant au centre de recherche Europes-Eurasie à l'INALCO. Ses travaux portent sur plusieurs domaines : linguistique, sociologie/anthropologie et histoire. Ancien élève de l'Institut d'études politiques de Paris et de l'Université Paris 1 Panthéon-Sorbonne, il a enseigné à l'Université pédagogique de Tachkent et travaillé comme adjoint au chef du département des relations internationales de l'Académie des sciences de l'Ouzbékistan. 